\title{
Deep learning based event reconstruction for Limadou HEPD
}

\section{Francesco Maria Follega, ${ }^{a, b, *}$ Marco Cristoforetti ${ }^{b, c}$ and Roberto luppa ${ }^{a, b}$ on behalf of the CSES-Limadou Collaboration}

(a complete list of authors can be found at the end of the proceedings)

\author{
${ }^{a}$ University of Trento, Dipartimento di Fisica, \\ Via Sommarive 14, 38123, Trento, Italy \\ ${ }^{b}$ Trento Institute for Fundamental Physcis and Applications (TIFPA), \\ Via Sommarive 14, 38123, Trento, Italy \\ ${ }^{c}$ Fondazione Bruno Kessler (FBK), \\ Via Sommarive 18, 38123, Trento, Italy \\ E-mail: francesco.follega@unitn.it, mcristofo@fbk.eu, \\ roberto.iuppa@unitn.it
}

Deep learning algorithms have gained importance in astroparticle physics in the last years. They have been shown to outperform traditional strategies in particle identification, tracking and energy reconstruction. The attractive feature of these techniques is their ability to model large dimensionality inputs and catch non-trivial correlations among the variables, which could be hidden or not easy to model. This contribution focuses on the application of deep neural networks to the event reconstruction of the Limadou High-Energy Particle Detector on board of the China Seismo-Electromagnetic Satellite. We describe the model adopted for the neural network and report on the performance measured on simulated and real data.

$37^{\text {th }}$ International Cosmic Ray Conference (ICRC 2021)

July 12th - 23rd, 2021

Online - Berlin, Germany

\footnotetext{
${ }^{*}$ Presenter
} 


\section{Event Reconstruction with Deep Learning}

The most modern particle physics and astrophysics experiments are increasingly including Deep Learning (DL) based models in their event reconstruction procedure [1, 2]. Such an approach has the main advantage that correlations between variables are discovered automatically by the algorithms via the minimization of the loss function in the learning procedure. These methods can help not only because physical intuition can miss some of these correlations, but also because the machine can automatically take into account detector asymmetries and data inhomogeneities to improve its prediction.

In this work we present an application of Deep Learning algorithms to space experiments. Two set of Fully Connected Neural Networks are designed to reconstruct particles traversing the Limadou High Energy Particle Detector (HEPD). The networks have as input the digitized signal from the detector and return as output the arrival direction in the local frame, the kinetic energy and the particle-type.

A large and reliable GEANT4 [3] simulation was produced to train and test the neural networks on data as close as possible to the real one. In particular the networks at the base of the reconstruction chain are trained on a sample of electrons with energy ranging between 1-200 MeV and a sample of protons with energy between $30-1000 \mathrm{MeV}$, simulated with an isotropic flux. Moreover, real test beam data are used to crosscheck the reconstruction performance.

\section{The CSES mission}

The Limadou High Energy Particle Detector is the Italian contribution to the China Seismo Electromagnetic Satellite (CSES) [4]. The CSES mission is a joint collaboration between the China National Space Agency (CNSA) and the Italian Space Agency (ASI) aiming to build a sophisticated space observatory focused on the monitoring of the electromagnetic environment, the plasma and particle populations in the atmosphere, ionosphere and magnetosphere. The main scientific objectives of the missions are:

- clarify on the existence and nature of a correlation between seismic events and variation of fluxes of charged particles in the Van Allen Belts;

- extend the previous measurements of low energy cosmic rays spectrum for protons and electrons [5], from few $\mathrm{MeV}$ to hundreds $\mathrm{MeV}$;

- study impulsive solar activity as Coronal Mass Ejections (CMEs) and solar flares.

On February 2018 the first satellite of the mission (CSES-01) equipped with nine instruments, among which there was Limadou HEPD, was launched and is now orbiting around the Earth. It is placed on a $98^{\circ}$ Sun-synchronous circular orbit at an altitude of about $500 \mathrm{~km}$, with an expected lifetime of at least 5 years. A second satellite will be launched by the end of 2022 to increase the coverage and the collected statistics. 


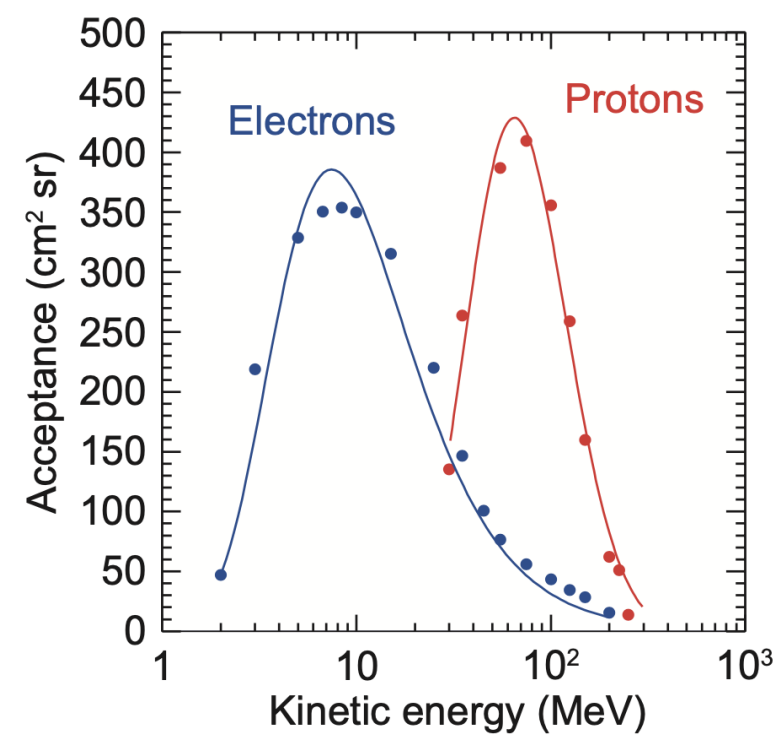

Figure 1: Estimated acceptances for simulated electrons and protons [4].

\section{Limadou HEPD: Detector and Simulation}

Limadou HEPD is optimized for the detection of 30-200 MeV protons and 3-100 MeV electrons (fig. 1). As shown in figure 2, it is composed by several submodules which performs different measurements:

- A tracker made of two planes of double-side silicon micro strip sensor planes placed on the top of the HEPD.;

- A trigger made of 6 paddles (200 mm x $30 \mathrm{~mm}$ x $5 \mathrm{~mm}$ each) of plastic scintillator, each one read out by a photomultiplier tube (PMT) on each side;

- A calorimeter divided in upper and lower modules. The upper calorimeter is made of 16 planes of plastic scintillator (150 mm x $150 \mathrm{~mm}$ x $10 \mathrm{~mm}$ ), each one read out by two PMTs. The lower calorimeter consists in an array of 3 x 3 matrix of LYSO crystals $(5 \mathrm{~mm}$ x $5 \mathrm{~mm}$ x $4 \mathrm{~mm}$ ), each one read out by a PMT;

- A veto system made of 5 planes of plastic scintillators that works as an anti-coincidence detector for particles not coming from the top of the apparatus.

This detector and the interaction with protons and electrons have been simulated using the GEANT4 toolkit [3]. The energy deposited in the scintillator planes is converted into light which is then collected by the PMTs. Indeed, the Monte Carlo simulation was tuned on data acquired during test beam runs before the launch [6]: a conversion factor from the number of photo-electrons in the scintillators and the LYSO crystals to ADC counts has been derived and applied to obtain exactly the signal produced by the detector. Events have been generated in the following phase space: 


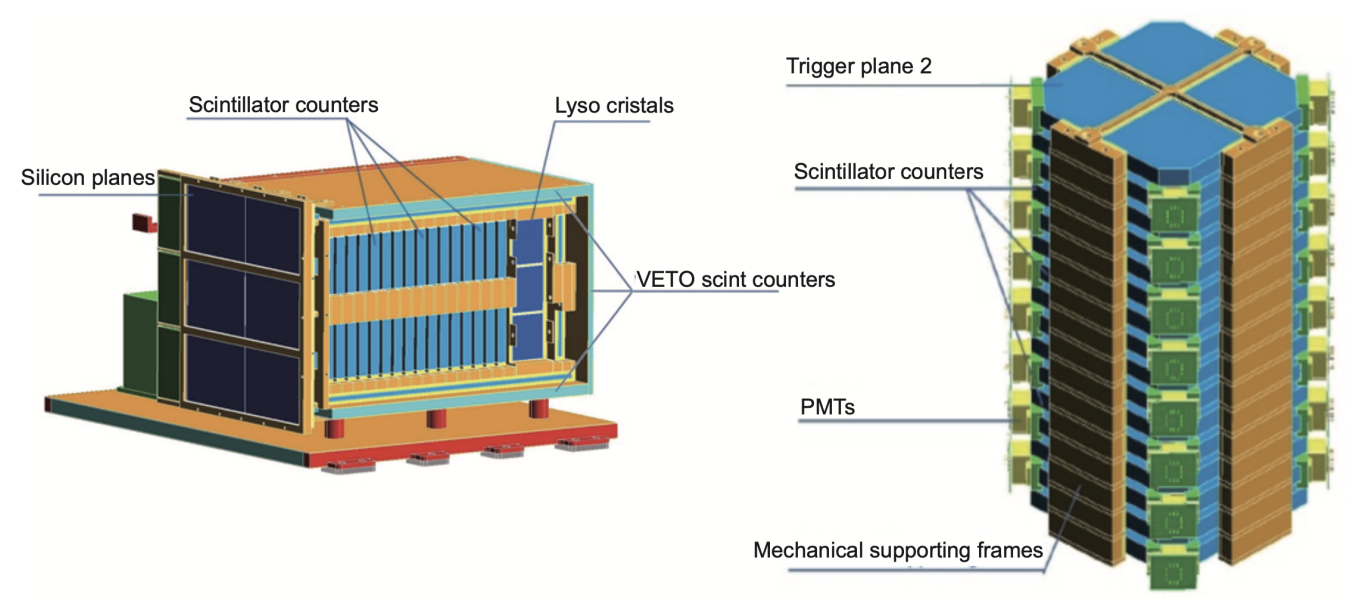

Figure 2: Estimated acceptances for simulated electrons and protons [4].

- isotropic flux: azimuthal angle $\phi$ sampled uniformly in $[-\pi, \pi]$ and polar angle $\theta$ sampled uniformly in $\cos ^{2} \theta$;

- Kinetic energy for electrons in $[0,200] \mathrm{MeV}$;

- Kinetic energy for protons in $[0,1000] \mathrm{MeV}$;

The event selection currently used with flight data was applied to train the FCNNs on the signal produced by passing trough particles:

$T \&(P 1 \& P 2) \&($ not LatVeto $)$

where $\mathrm{T}$ means a signal on at least one of the trigger paddles, P1 and P2 mean that we require a signal on the first and second plane and "not LatVeto" stays for no signal registered by the lateral veto planes.

\section{DL-based reconstruction strategy}

A sketch of the reconstruction scheme is shown in fig. 3. The input set is represented by a vector of 53 signals produced by the particle interaction with the detector: 12 signals of the trigger PMTs, the 32 signals coming from the upper calorimeter PMTs and the 9 signals from the LYSO crystals. These signals are used to train two sets of Fully Connected Neural Networks with a similar structure, 4 hidden layers with up to 512 nodes. The first one discriminates between electrons and protons (classification task - BCELoss [7]). The second ones reconstructs the kinetic energy and the arrival direction of the incoming particle (regression task - L1Loss [8] + angular distance).

The NNs are designed and trained using PyTorch [9]. They were trained keeping the $80 \%$ of the Monte Carlo dataset for the training and validation sets and the remaining $20 \%$ as independent test set on which we evaluate the performance. 


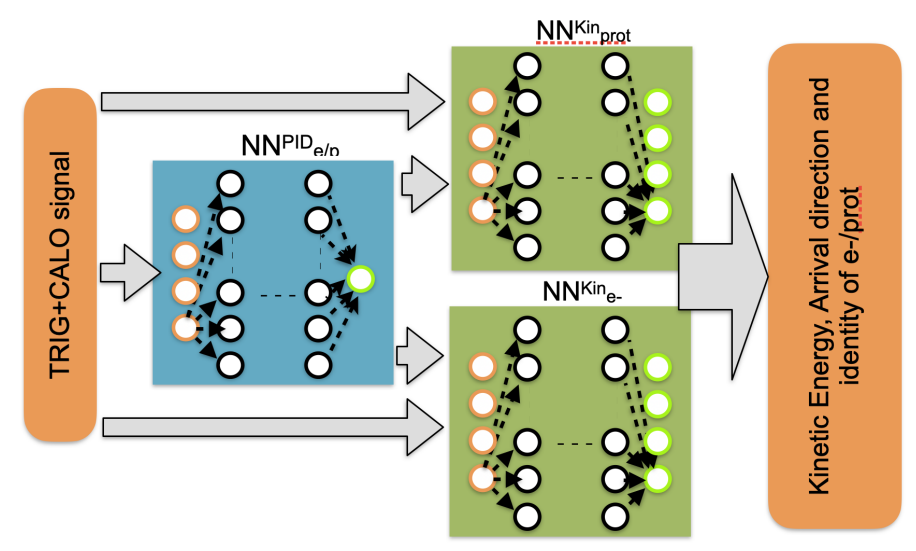

Figure 3: A sketch of the DL-based reconstruction scheme is shown in the picture.
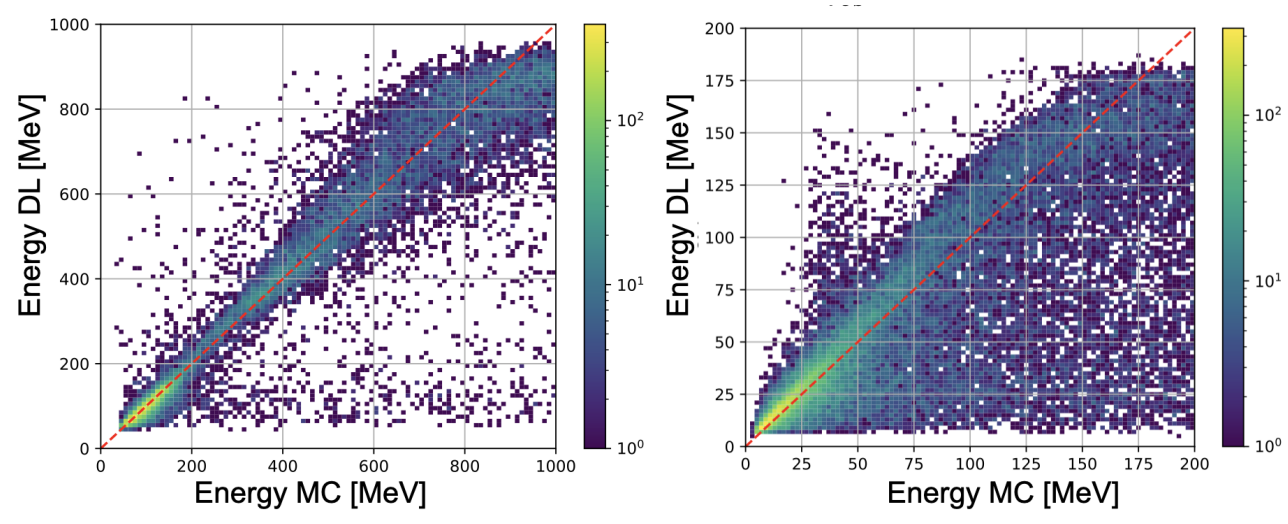

Figure 4: The kinetic energy generated with our MC vs. the one estimated with DL-based algorithms for protons (left) and electrons (right). The dashed red line is the bisector.

\section{Performance on Monte Carlo and results on Test Beam data}

Protons and electrons are discriminated by the first NN with a cumulative accuracy of $97.1 \%$ over all the simulated energy ranges. The particle identification performance is particularly challenging at higher energies when particles are not contained anymore and the shape of their energy deposits in the detector start to be similar.

The performance of the NNs in the reconstruction of the kinetic energy from the ADC signals are quite different in the case of protons and electrons. This was expected due to different ways with which the two particles release their energy on the scintillators and it was one of the main the reason for training two separate networks. In fig. 4-left we can appreciate how the protons $\mathrm{FCNN}_{K I N}$ is able to correctly predict the energy in the range $50-1000 \mathrm{MeV}$, with some saturation effects that emerge after that point up to $800 \mathrm{MeV}$. The DL-based algorithms are able to reconstruct protons not fully contained in the calorimeter (full containment around $200 \mathrm{MeV}$ for protons), looking the raising of the charge deposition plane-by-plane. On the other hand, this is not true for the electrons (fig. 4-right) where the reconstruction is heavily affected by containment issues and the energy lost via Bremsstrahlung, which is not measured in the scintillators planes/LYSO crystals.

The arrival direction is estimated at the same time of the kinetic energy. The DL-based 

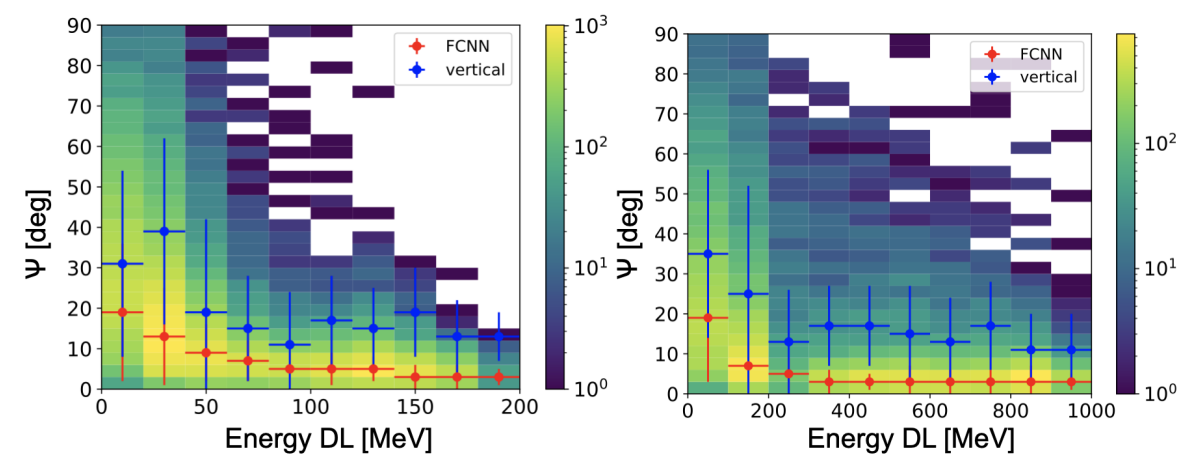

Figure 5: The angular difference $\Psi$ as a function of the kinetic energy reconstructed by our neural networks, for electrons (left) and protons (right). The red points represents the error in the reconstruction with DL approach, the blue points the one obtained with the vertical pointing. In both cases the error bars are the half width at half maximum of the distributions.
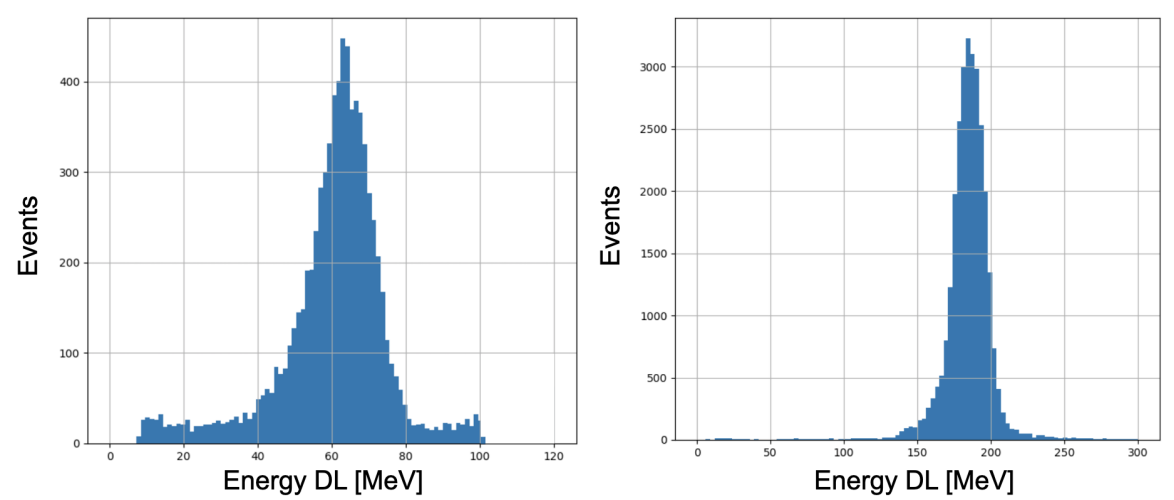

Figure 6

reconstruction strategy exploits two things: the correlation between the difference in the charge collected by the 2 PMTs of the same plane along all the planes and the correlation between the arrival direction and kinetic energy. In fig. 5 is shown the behaviour of the angular difference between the true and reconstructed direction as a function of the energy. Obviously, the more energetic is the particle the more it penetrates inside the calorimeter producing more signal to be fed to our DL-based reconstruction chain and therefore the performances improve. As usual, the angular resolution is better for protons which lose more energy in the calorimeter, producing more information about their trajectory. In both cases the direction provided by the NNs is better then the one obtained pointing always the vertical $(\theta=0)$.

The same neural networks have been applied also to the test beam data used to calibrate the apparatus before the launch [6]. In fig. 6, we show an example of the performance in the kinetic energy reconstruction for $60 \mathrm{MeV}$ electrons acquired at the Beam Test Facility of Frascati (Italy) and for $174 \mathrm{MeV}$ (protons) acquired at the APSS Proton Therapy of Trento (Italy).

The energy is reconstructed correctly for electrons and protons within (5\%). A slight overestimation is present due to Data/MC differences and will be corrected with an unfolding procedure. 


\section{Conclusions}

In this contribution is shown how a Deep Learning based approach to the event reconstruction can be applied successfully to the case of the Limadou HEPD. The DL chain is able to reconstruct the particle identity, the kinetic energy and the arrival direction of the incoming particle. DL efficiently reconstruct the kinetic energy also for not contained protons that cannot be obtained with standard methods. These algorithms show good performance and has been adopted by the collaboration for the reconstruction of real data acquired in flight.

\section{References}

[1] D. Guest, K. Cranmer, and Daniel Whiteson, Performance of b-jet identification in the ATLAS experiment, Annu. Rev. Nucl. Part. Sci. 68 (2018)

[2] The CMS Collaboration, A deep neural network for simultaneous estimation of b jet energy and resolution, CERN-EP-2019-261 (2019)

[3] S.Agostinelli et al., Geant4 simulation toolkit, Nuclear Instruments and Methods in Physics Research A 506250 (2003)

[4] P. Picozza et al. The HEPD particle detector of the CSES satellite mission for investigating seismo-associated perturbations of the Van Allen belts, J. Phys.: Conf. Ser. 798012033 (2017)

[5] A. M. Galper et al. The PAMELA experiment: a decade of Cosmic Ray Physics in space, J. Phys.: Conf. Ser. 798012033 (2017)

[6] P. Picozza et al. Beam test calibrations of the HEPD detector on board the China SeismoElectromagnetic Satellite, Nuclear Inst. and Methods in Physics Research, A 974 (2020) 164170

[7] Pytorch implementation of BCE, https://pytorch.org/docs/stable/generated/torch.nn.BCELoss.html

[8] Pytorch implementation of L1Loss, https://pytorch.org/docs/stable/generated/torch.nn.L1Loss.html

[9] A. Paszke et al. PyTorch: An Imperative Style, High-Performance Deep Learning Library, Advances in Neural Information Processing Systems 32 (2019) 


\section{Full Authors List: CSES-Limadou Collaboration}

S. Bartocci ${ }^{1}$, R. Battiston ${ }^{2,3}$, F. Benotto ${ }^{4}$, S. Beolé, ${ }^{4,5}$, W.J. Burger ${ }^{3,6}$, D. Campana ${ }^{7}$, G. Castellini ${ }^{8}$, P. Cipollone ${ }^{1}$, S. Coli ${ }^{4}$, L. Conti ${ }^{1,9}$, A. Contin ${ }^{10,11}$, M. Cristoforetti ${ }^{12}$, L. De Cilladi ${ }^{4,5}$, C. De

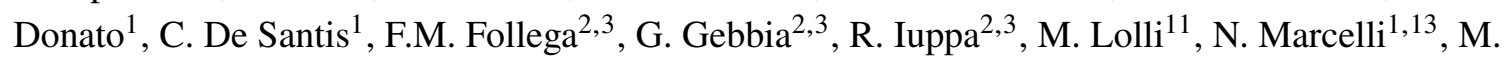
Martucci $^{1,13}$, G. Masciantonio ${ }^{1}$, M. Mergé ${ }^{1, \dagger}$, M. Mese ${ }^{7,14}$, C. Neubuser ${ }^{3}$, F. Nozzoli ${ }^{3}$, A. Oliva ${ }^{11}$,

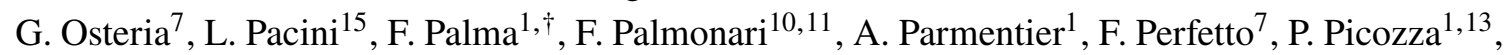
M. Piersanti ${ }^{16}$, M. Pozzato $^{11}$, E. Ricci ${ }^{2,3}$, M. Ricci ${ }^{17}$, S.B. Ricciarini ${ }^{8}$, Z. Sahnoun ${ }^{11}$, V. Scotti ${ }^{71}$, A. Sotgiu ${ }^{1,13}$, R. Sparvoli ${ }^{1,13}$, V. Vitale ${ }^{1}$, S. Zoffoli $^{18}$ and P. Zuccon ${ }^{2,3}$

${ }^{1}$ INFN-Sezione di Roma “Tor Vergata”, V. della Ricerca Scientifica 1, I-00133 Rome, Italy;

${ }^{2}$ University of Trento, V. Sommarive 14, I-38123 Povo (Trento), Italy;

${ }^{3}$ INFN-TIFPA, V. Sommarive 14, I-38123 Povo (Trento), Italy;

${ }^{4}$ INFN-Sezione di Torino, Via P. Giuria 1, I-10125 Torino, Italy;

${ }^{5}$ University of Torino, Via P. Giuria 1, I-10125 Torino, Italy;

${ }^{6}$ Centro Fermi, V. Panisperna 89a, I-00184 Rome, Italy;

${ }^{7}$ INFN-Sezione di Napoli, V. Cintia, I-80126 Naples, Italy;

${ }^{8}$ IFAC-CNR, V. Madonna del Piano 10, I-50019 Sesto Fiorentino (Florence), Italy;

${ }^{9}$ Uninettuno University, C.so V. Emanuele II 39, I-00186 Rome, Italy;

${ }^{10}$ University of Bologna, V.le C. Berti Pichat 6/2, I-40127 Bologna, Italy;

${ }^{11}$ INFN-Sezione di Bologna, V.le C. Berti Pichat 6/2, I-40127 Bologna, Italy;

${ }^{12}$ Fondazione Bruno Kessler, V. Sommarive 18, I-38123 Povo (Trento), Italy;

${ }^{13}$ University of Rome "Tor Vergata”, V. della Ricerca Scientifica 1, I-00133 Rome, Italy;

${ }^{14}$ University of Naples "Federico II", V. Cintia 21, I-80126 Naples, Italy;

15 INFN-Sezione di Firenze, V. Sansone 1, I-50019 Sesto Fiorentino (Florence), Italy;

${ }^{16}$ INAF-IAPS, V. Fosso del Cavaliere 100, I-00133 Rome, Italy;

${ }^{17}$ INFN-LNF, V. E. Fermi 54, I-00044 Frascati (Rome), Italy;

${ }^{18}$ Italian Space Agency, V. del Politecnico, I-00133 Rome, Italy;

$\dagger$ At ASI Space Science Data Center (SSDC) also, V. del Politecnico, I-00133 Rome, Italy. 